九州大学学術情報リポジトリ

Kyushu University Institutional Repository

\title{
Phasing Trajectories to Deploy a Constellation in a Halo Orbit
}

Chen, Hongru

Key Laboratory of Space Utilization, Technology and Engineering Center for Space Utilization, Chinese Academy of Sciences

Ma, Jian

Key Laboratory of Space Utilization, Technology and Engineering Center for Space Utilization, Chinese Academy of Sciences

http://hdl. hand le. net/2324/4481566

出版情報: Journal of Guidance, Control, and Dynamics. 40 (10), pp.2662-2667，2017-10. American Institute of Aeronautics and Astronautics

バージョン :

権利関係 : 


\title{
Phasing Trajectories to Deploy a Constellation in a Halo Orbit
}

\author{
Hongru Chen ${ }^{*}, 1$ and Jian $\mathrm{Ma}^{1,2}$ \\ ${ }^{1}$ Key Laboratory of Space Utilization, Technology and Engineering Center for Space Utilization, Chinese Academy \\ of Sciences, Beijing, China 100094 \\ ${ }^{2}$ University of Chinese Academy of Sciences
}

\section{Introduction}

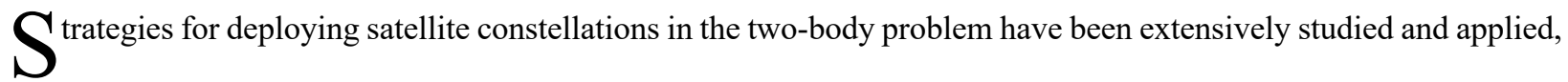
whereas the present Note concerns deploying a constellation in the three-body problem. Motivated by a proposed CubeSat project PHOEBE (Positioning using earth-moon Halo Orbit Experimental BEacons) for positioning the users on the far side of Moon [1], this study investigates methods of designing the phasing trajectory to deploy a constellation in a halo orbit with low $\Delta v$. The halo orbit is a three-dimensional periodic orbit about a libration point in the three-body system.

There will be missions aimed at the far side of the Moon in the near future [2]. The terrain and resources on the far side of the Moon are very different from those on the near side. In addition, the far side is an ideal platform for radio astronomy since the Moon can shield radio noises from the Earth. China will land the Chang'E-4 on the far side in 2018 [3]. However, challenges for navigation and communication arise, as the far side is invisible to the Earth. Moreover, as the terrain on the far side is very rough, the requirement of landing accuracy is even strict. Hill and Born [4], and Hesar et al. [5] have studied the technique of tracking the satellites in an Earth-Moon L2 (EML2) halo orbit and landers on the far side of Moon using inter-satellite ranging. However, that technique is applicable for nonmaneuvering objects. For real-time positioning of maneuvering objects, such as the lander performing pinpoint landing, multiple reference points are required. Referring to the GPS technique, PHOEBE involves four CubeSats in an EML2 halo orbit to provide the references. The averages are:

1) EML2 halo orbits can always be seen from the Earth and the far side of the Moon, which is favorable for constant communication as well as tracking.

\footnotetext{
${ }^{*}$ Assistant Researcher, 9 Dengzhuang S Rd, hongru.chen@hotmail.com
} 
2) CubeSats are miniature and fully functional, and thus can be easily carried by a mother spacecraft along with a lander going to the Moon.

For instance, ESA's AIM mission is planned to rendezvous the binary asteroid 65803 Didymos and then deploy a lander and two or more CubeSats to perform inter-satellite network [6]. It is reasonable to believe that deploying CubeSats to perform cooperative operations is a promising manner in future space missions.

To support positioning service, it is required that the four CubeSats form a favorable configuration. The limited propulsion capability of CubeSats significantly constrains trajectory options. There have been many studies (e.g. Refs. [7-9]) on the design of low- $\Delta v$ transfer trajectories based on the invariant manifold associated with libration-point orbits. Folta et al [10] and Mathur [11] have studied the transfer trajectories suitable for lunar CubeSats. However, to form a constellation, it is not economical to launch the CubeSats one by one. This work applies the three-body dynamics to the design of deployment trajectories.

This Note is organized as follows. Sec. II briefly presents the model of the circular restricted three-body problem, halo orbit and invariant manifold. Sec. III gives an overview of the PHOEBE mission, including the requirement for deploying the CubeSats along a halo orbit as evenly as possible. Sec. IV discusses the methods of designing phasing trajectories for deployment. Three approaches, namely, two-impulsive correction maneuvers, patched manifolds and combination of manifolds and symmetric connecting trajectories, are presented. Conclusions are given in Sec. V.

\section{System Dynamics}

\section{A. Equations of Motion}

Trajectories are considered in the Earth-Moon circular restricted three-body problem (CR3BP). The CR3BP assumes that two primary bodies $m_{1}$ and $m_{2}$ are moving in a circular orbit about their barycenter. The mass of the third body is negligible compared to the masses of the two primaries. The rotating coordinate system with the origin at the barycenter, $x$ axis along the vector between the primaries, and the $z$ axis parallel to the rotating direction of the primary system is chosen to describe the motion of the third body. For convenience, the model is normalized by setting the angular velocity of the rotating frame, the sum of the masses of the primaries, and the distance between the two primaries to be units. Let LU represent the length unit and TU the time unit in this system. $\mu$ is the ratio of the mass of $m_{2}$ to the mass of $m_{1}$ ( $\mu=0.0122$ for the Earth-Moon system). Then, masses of $m_{1}$ and $m_{2}$ become $1-\mu$ and $\mu$ in the 
normalized model. The coordinates of $m_{1}$ and $m_{2}$ become $[-\mu, 0,0]$ and $[1-\mu, 0,0]$, respectively, as depicted in Fig. 1. The equations of motion of the third body are:

$$
\begin{gathered}
\ddot{x}-2 \dot{y}=\partial U / \partial x \\
\ddot{y}+2 \dot{x}=\partial U / \partial y \\
\ddot{z}=\partial U / \partial z
\end{gathered}
$$

where the gravitational potential $U$ in the system is

$$
U=\left(x^{2}+y^{2}\right) / 2+(1-\mu) / r_{1}+\mu / r_{2}
$$

where

$$
r_{1}=\sqrt{(x+\mu)^{2}+y^{2}+z^{2}}, r_{2}=\sqrt{(x-1+\mu)^{2}+y^{2}+z^{2}}
$$

In addition, Eqs. (1)- (3) indicate a symmetry about the $x$-z plane. To be specific, if a set $(x, y, z, \dot{x}, \dot{y}, \dot{z}, \ddot{x}, \ddot{y}, \ddot{z}, t)$ is a solution to the equations of motion, the set $(x,-y, z,-\dot{x}, \dot{y},-\dot{z}, \ddot{x},-\ddot{y}, \ddot{z},-t)$ is also a solution.

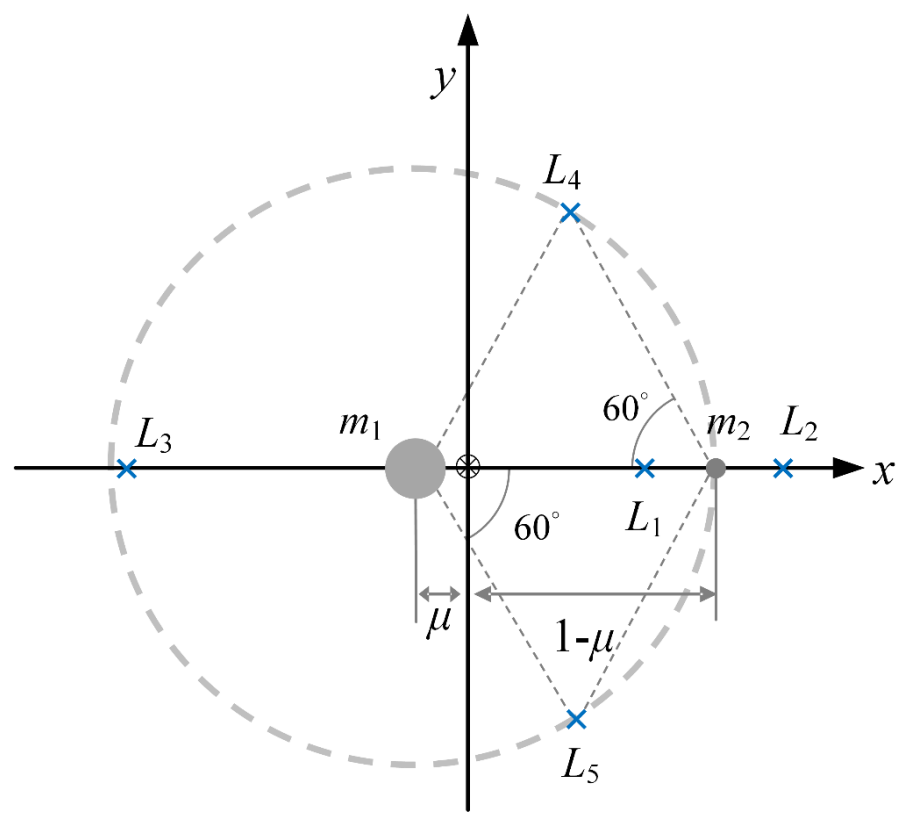

Fig. 1. Rotating frame of CR3BP and equilibrium points $L_{1}, L_{2}, \ldots, L_{5}$. 


\section{B. Halo Orbits and Invariant Manifolds}

The CR3PB has five equilibrium points where velocity and acceleration are zero. They are also termed libration points or Lagrangian points labeled as $L_{1}, \ldots, L_{5}$. Their geometries are depicted in Fig. 1 (for details of resolving the equilibrium points, see Ref. [12]). The halo orbit is a three-dimensional periodic orbit around a collinear point, $L_{1}, L_{2}$, or $L_{3}$. Halo orbits can be computed using numerical differential correction [13]. Fig. 2 shows a group of EML2 halo orbits. The period of an Earth-Moon $L_{1} / L_{2}$ halo orbit is around 15 days.
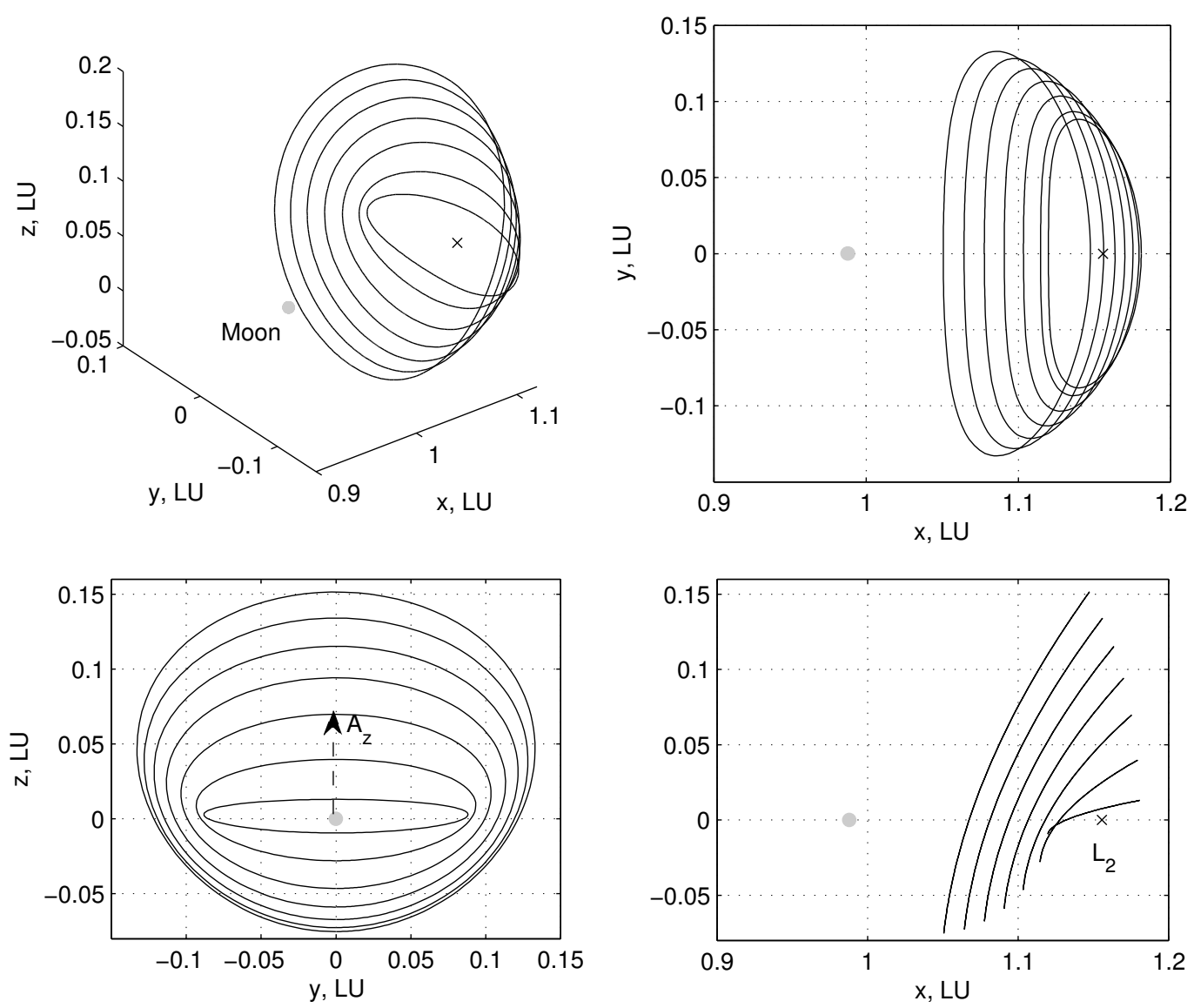

Fig. 2. Earth-Moon $L_{2}$ halo orbits.

There are a stable invariant manifold and an unstable invariant manifold associated with a halo orbit. Invariant manifolds are generated based on the eigenvectors of the monodromy matrix. The monodromy matrix is the state transition matrix for one period of a periodic orbit. For a halo orbit, it has a pair of eigenvalues $\lambda_{1}$ and $\lambda_{2}$ with $\lambda_{1}>1$, $\lambda_{2}<1$, and $\lambda_{1} \cdot \lambda_{2}=1$. If a small displacement is placed along the eigenvector of the eigenvalue $\lambda_{1}$, it will grow exponentially downstream. The resulting divergent trajectory is along the unstable manifold associated with the halo orbit. Conversely, backward propagating the states perturbed in the eigenvector of $\lambda_{2}$ yields a stable manifold that 
asymptotically converges into the halo orbit. Fig. 3 shows the stable and unstable manifold trajectories derived from the Earth-Moon $L_{2}$ halo orbit with z-amplitude $A_{z}=15,000 \mathrm{~km}$.

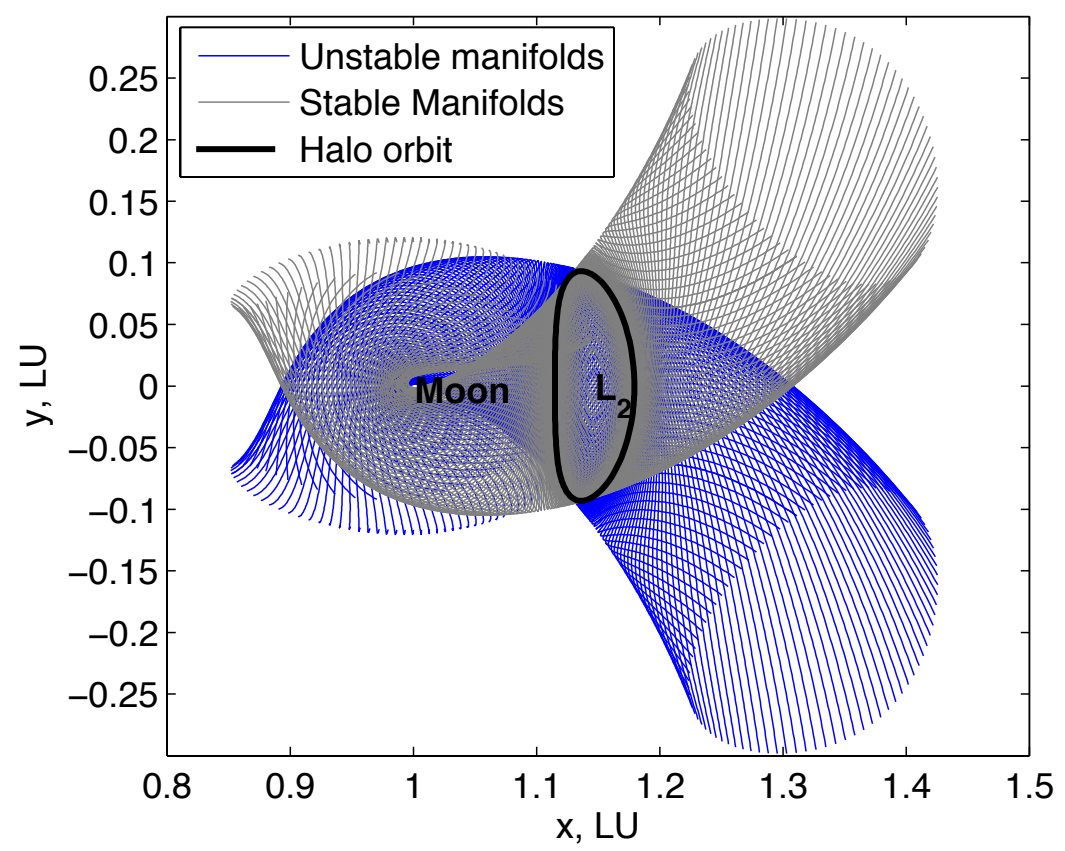

Fig. 3. An EML2 halo orbit and the stable and unstable manifolds associated with it.

\section{Parameterization}

Invariant manifolds can be used as the pathway to send spacecraft from or to the halo orbit at little $\Delta v \operatorname{cost}$. In this work, invariant manifolds instead of the halo orbit are the initial and final conditions of the trajectory design. For the concerned optimization problem, the manifold and halo orbit should be parameterized. Let $T$ denote the period of the halo orbit, and $\tau(0 \leq \tau<T)$ denote the time past a reference point in the halo orbit. The $x-z$ plane crossing on the far side was taken as the reference point for this work. $\Delta \mathbf{v}$ are added at different points defined by $\tau$ to generate manifold trajectories. The $\Delta \mathbf{v}$ direction is chosen to be equivalent to the divergent eigenvector or convergent eigenvector (for details, see Ref. [14]). Magnitudes of departure and insertion $\Delta \mathbf{v}$ are all set to be $1 \mathrm{~cm} / \mathrm{s}$. Then, the manifold trajectory can be uniquely specified by $\tau$. 


\section{III.Problem Statement}

The EML2 is $64,500 \mathrm{~km}$ from the Moon. The halo orbits around EML2 are visible to both the Earth and the far side of the Moon. Moreover, the directions of the lines of sight to the Earth and the Moon are almost aligned, which is favorable for communication as well as attitude control. The PHOEBE project is to deploy four 6U CubeSats along an EML2 halo orbit to support the positioning of users on the far side of the Moon (see Fig. 4).

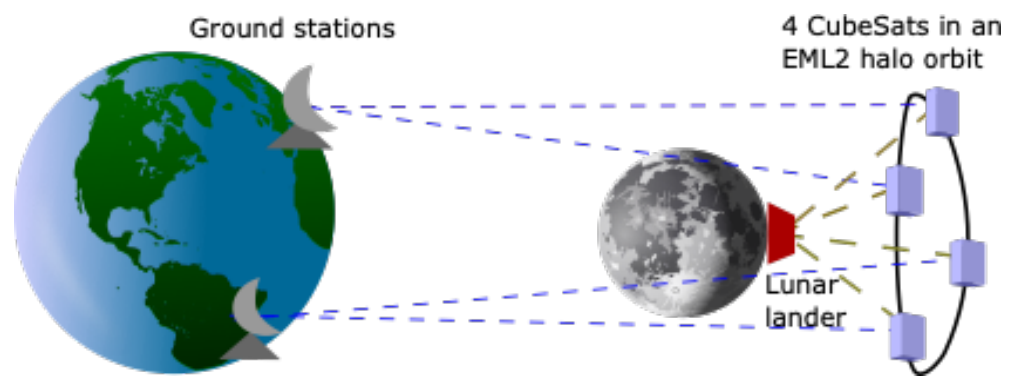

Fig. 4. Schematic of tracking CubeSats and lunar landers on the far side.

The positions of the CubeSats are determined by processing the ranging data from the ground stations on Earth. With the positions of the CubeSats known, through ranging between the user on the far side of the Moon and the CubeSats, the user can resolve its position through a proper estimation algorithm. The position error $(\Delta$ position $)$ of the user can be expressed as the product of the pseudorange error (Apseudorange) and a geometric index; that is,

$$
\Delta \text { position }=\text { GDOP } \times \Delta \text { pseudorange }
$$

where GDOP (Geometric Dilution of Precision) is related to the configuration of the CubeSats with respect to the user. GDOP can be computed from,

$$
\mathbf{H}=\left[\begin{array}{cccc}
\frac{\left(x_{1}-x\right)}{R_{1}} & \frac{\left(y_{1}-y\right)}{R_{1}} & \frac{\left(z_{1}-z\right)}{R_{1}} & -1 \\
\frac{\left(x_{2}-x\right)}{R_{2}} & \frac{\left(y_{2}-y\right)}{R_{2}} & \frac{\left(z_{2}-z\right)}{R_{3}} & -1 \\
\frac{\left(x_{3}-x\right)}{R_{3}} & \frac{\left(y_{3}-y\right)}{R_{3}} & \frac{\left(z_{3}-z\right)}{R_{3}} & -1 \\
\frac{\left(x_{4}-x\right)}{R_{4}} & \frac{\left(y_{4}-y\right)}{R_{4}} & \frac{\left(z_{4}-z\right)}{R_{4}} & -1
\end{array}\right]
$$


where $x, y$ and $z$ denote the position of the user, $x_{\mathrm{i}}, y_{\mathrm{i}}$ and $z_{\mathrm{i}}$ denote the position of the $i$-th CubeSat, and $R_{i}$ denotes the distance between the user and the $i$-th CubeSat. The smaller the GDOP is, the better the configuration is for positioning. With no influence of atmosphere on the Moon, spseudorange is considered mainly contributed by the error of orbit determination of the CubeSats. According to orbit determination result of ARTEMIS [15], $\Delta$ pseudorange is assumed to be 20 meters. For a desirable $\Delta$ position of $1 \mathrm{~km}$, GDOP should be smaller than 50 .

GDOP depends on the configuration of the CubeSats. In this note, the interval between two CubeSats is represented by the difference of their phase angles, which is,

$$
\Delta \varphi=\Delta \tau / T \times 360^{\circ}
$$

where $\Delta \tau$ is the difference between the $\tau$ of two CubeSats in the halo orbit. Then, $\Delta \varphi$ is associated with $\Delta \tau$. Fig. 4 shows the configurations with different $\Delta \varphi$ between neighboring CubeSats and different $A_{z}$ of halo orbits, and the corresponding GDOP in a period. The GDOP for the same $\Delta \varphi$ does not change too much with $A_{\mathrm{z}}$. However, GDOP is greatly depending on $\Delta \varphi$. Without doubt, the best configuration is that they are evenly spaced along the halo orbit; namely, they are separated evenly by a phase angle difference $\Delta \varphi=90^{\circ}$. As indicated in the figure, the GDOP $<50$ holds for $86 \%$ of the time for this case, which means the positioning performance is satisfactory for $86 \%$ of the time. In addition, a working interval lasts 3.2 days, and a blackout interval lasts 0.5 day. As the period of a low lunar orbit (e.g. 2 hours for an orbit with an altitude of $100 \mathrm{~km}$ ) and duration of landing (i.e. around $12 \mathrm{~min}$ ) are comparatively short, the time waiting for a landing is not be longer than 0.5 day.

On the other hand, it is not economical to launch four CubeSats separately. Therefore, it is assumed that a mother spacecraft carries a lunar lander and four CubeSats to the Moon. In the transfer phase from the Earth to the halo orbit, the rocket and mother spacecraft can provide the required powerful boosts. However, it is infeasible for the mother spacecraft to bring four CubeSats to their different destinations one by one, as the fuel cost will be unacceptably high. Therefore, it is assumed that the CubeSats as a whole are released at the stable manifold heading for the halo orbit at once. Then, the CubeSats are distributed into different phase angles of the halo orbit using their propulsion systems. Constrained by size, CubeSats have limited propulsion capacity. Therefore, it is needed to design the phasing trajectories that require affordable $\Delta v$. 

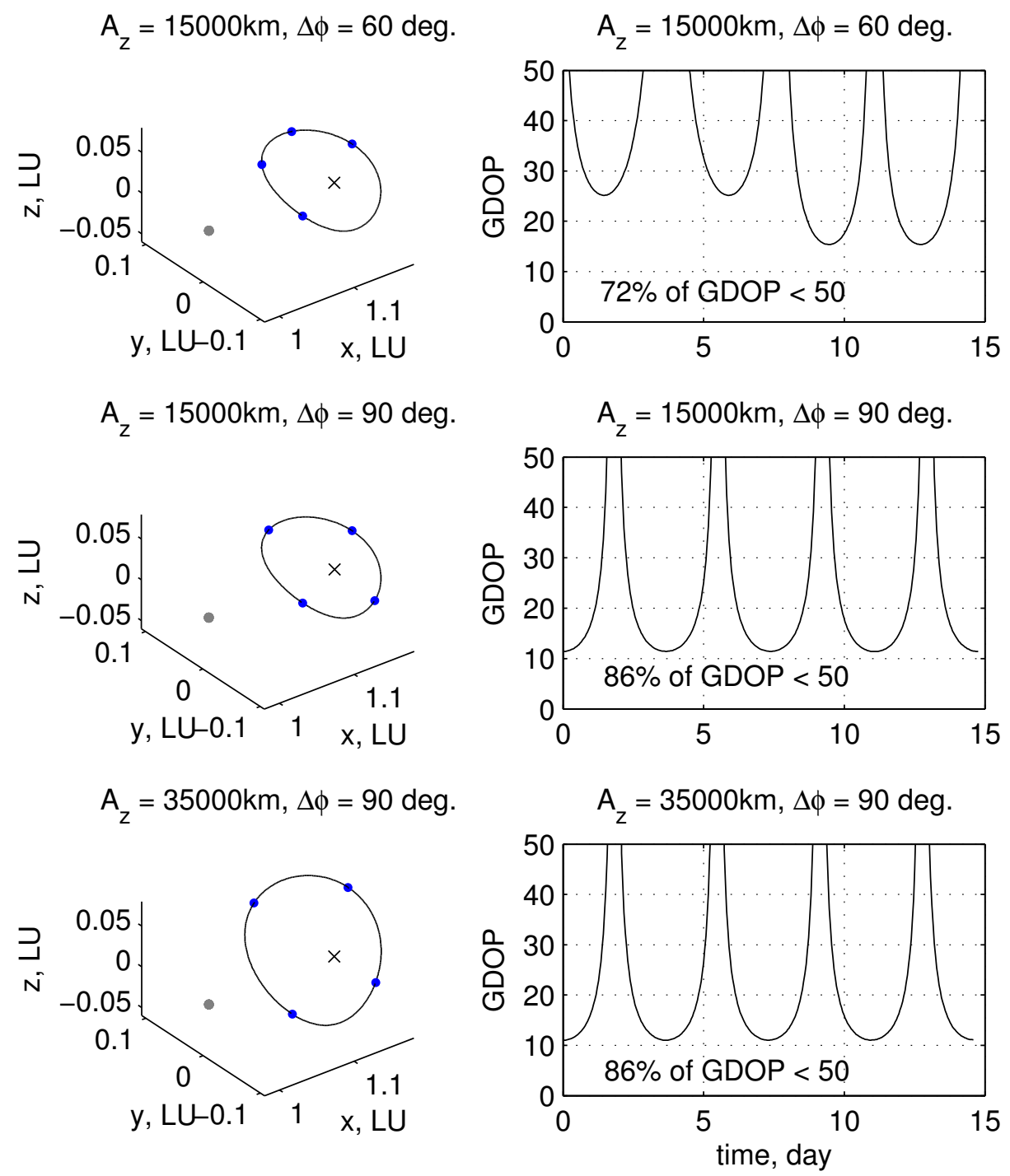

Fig. 5. Different configurations and the corresponding GDOP.

\section{IV.Phasing Trajectory Design}

\section{a. Two-impulsive Correction Maneuvers}

The halo orbit with $A_{z}=15,000 \mathrm{~km}$ is taken for the case study hereafter. The CubeSats are released at an initial stable manifold trajectory associated with the halo orbit. Applying maneuvers can send the CubeSats into another stable manifold trajectory whose $\tau$ is $\Delta \tau$ from the initial $\tau_{0}$. The two-impulsive correction maneuver is the most common way to correct a trajectory. The first maneuver $\Delta v_{1}$ is to deliver the CubeSat to meet a position along the target manifold trajectory, and the second maneuver $\Delta v_{2}$ is to cancel the velocity mismatch at the patch point. In summary, for a specified $\Delta \varphi$, the optimization program is to solve for the independent variables, namely, $\tau_{0}$, departure 
epoch, $t_{1}, \Delta \mathbf{v}_{1}$ at $t_{1}$, arrival epoch $t_{2}$, and $\Delta \mathbf{v}_{2}$ at $t_{2}$, such that the trajectory state meets the target trajectory state after $t_{2}$ and the total $\Delta v$ (i.e. $\Delta v_{1}+\Delta v_{2}$ ) is a minimum. Optimization is done with the MATLAB fmincon routine. Multiple initial guesses of $t_{1}$ and $t_{2}$ are tried.

Table 1 lists the minimum $\Delta v$ for various $\Delta \varphi$. Fig. 6 shows an example of changing the manifold trajectory to the one (blue) with a $\Delta \varphi$ of $-120^{\circ}$ from the initial (black). To form the best configuration, it is necessary to acquire a $\Delta \varphi$ of $+180^{\circ}$ or $-180^{\circ}$. However, the required $\Delta v$ of $240 \mathrm{~m} / \mathrm{s}$ is far beyond the capacity of a CubeSat.

Table 1. Minimum $\Delta v$ for different $\Delta \varphi$ using two-impulsive correction maneuvers.

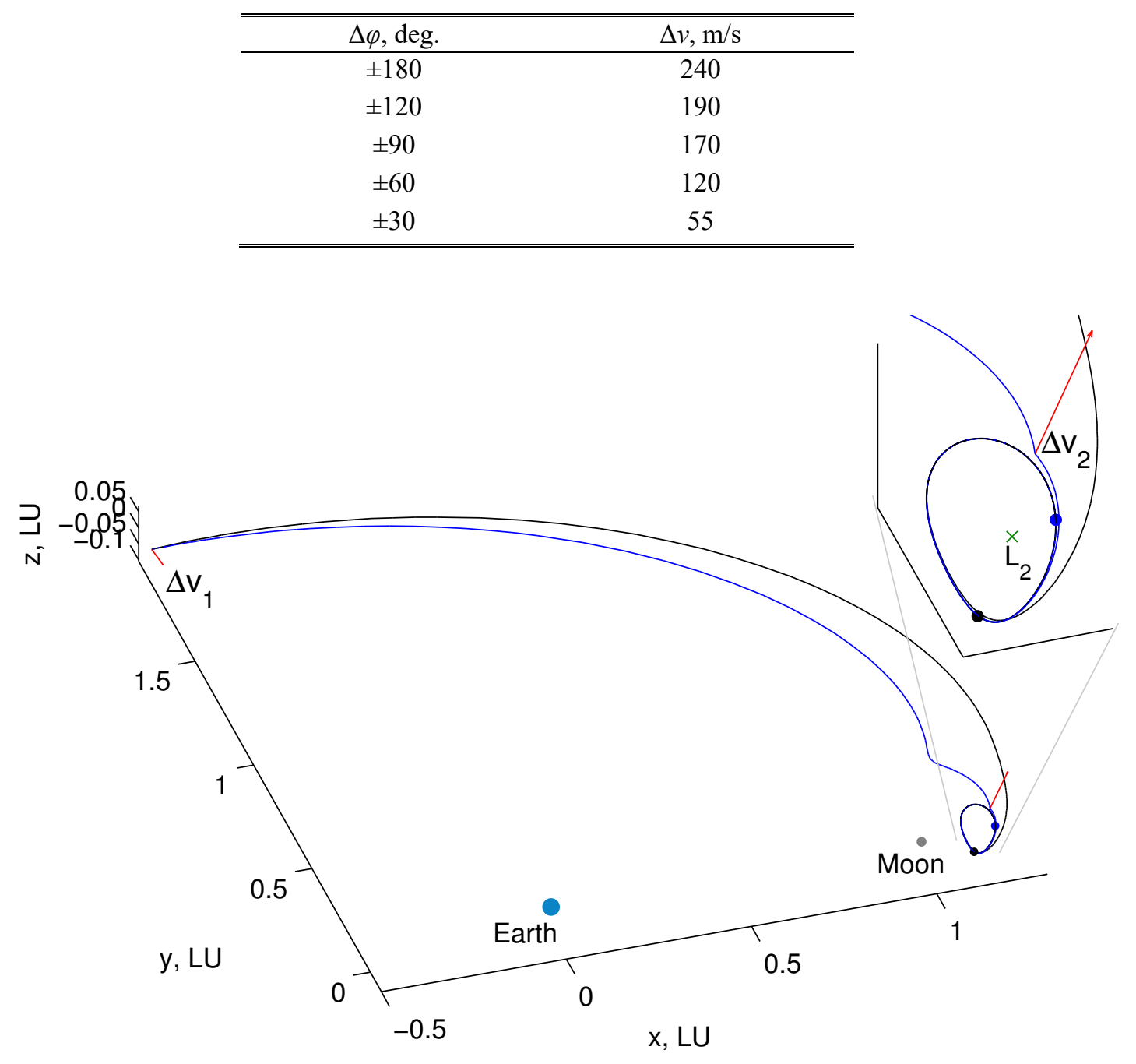

Fig. 6. Two-impulsive correction maneuvers (arrow) to cause a $\Delta \varphi$ of $-120 \mathrm{deg}$. 


\section{b. Patched Manifolds}

By observing Fig. 3, one can notice that the Moon-ward manifolds are turned by the lunar gravity when close to the Moon. Recalling the symmetry of the CR3BP, the unstable manifold and stable manifold completely match at $y=$ 0 in position (see Fig. 2). However, the velocity difference at $y=0$ is not zero. If a $\Delta v$ is paid to cancel that velocity mismatch, the CubeSat can go through the unstable and stable manifolds, and back in the halo orbit resulting in a $\Delta \varphi$.

According to the symmetry, $\Delta v=2 \sqrt{v_{x}^{2}+v_{z}^{2}}$, where $v_{x}$ and $v_{z}$ are the $x$ - and $z$-components of the velocity of the manifolds crossing the $x-z$ plane, respectively. $\Delta \varphi$ is due to the additional time spent on the transfer along the manifolds. Let $\tau_{0}$ represents the $\tau$ of the departure manifold trajectory. Then, $T-\tau_{0}$ is the $\tau$ of the insertion manifold trajectory because of the symmetry. $\Delta \varphi$ is computed from,

$$
\Delta \varphi=\operatorname{rem}\left(T-2 \tau_{0}-T o F, T\right) / T \times 360^{\circ}
$$

where $T o F$ is the flight time from leaving the halo orbit to insertion to the halo orbit. As new phase angles lag the initial phase angle, negative values are used for $\Delta \varphi$. Fig. 7 displays the manifolds on the Poincare section of $y=0$, along with the corresponding $\Delta v$ and $\Delta \varphi$ measured by the color scale.

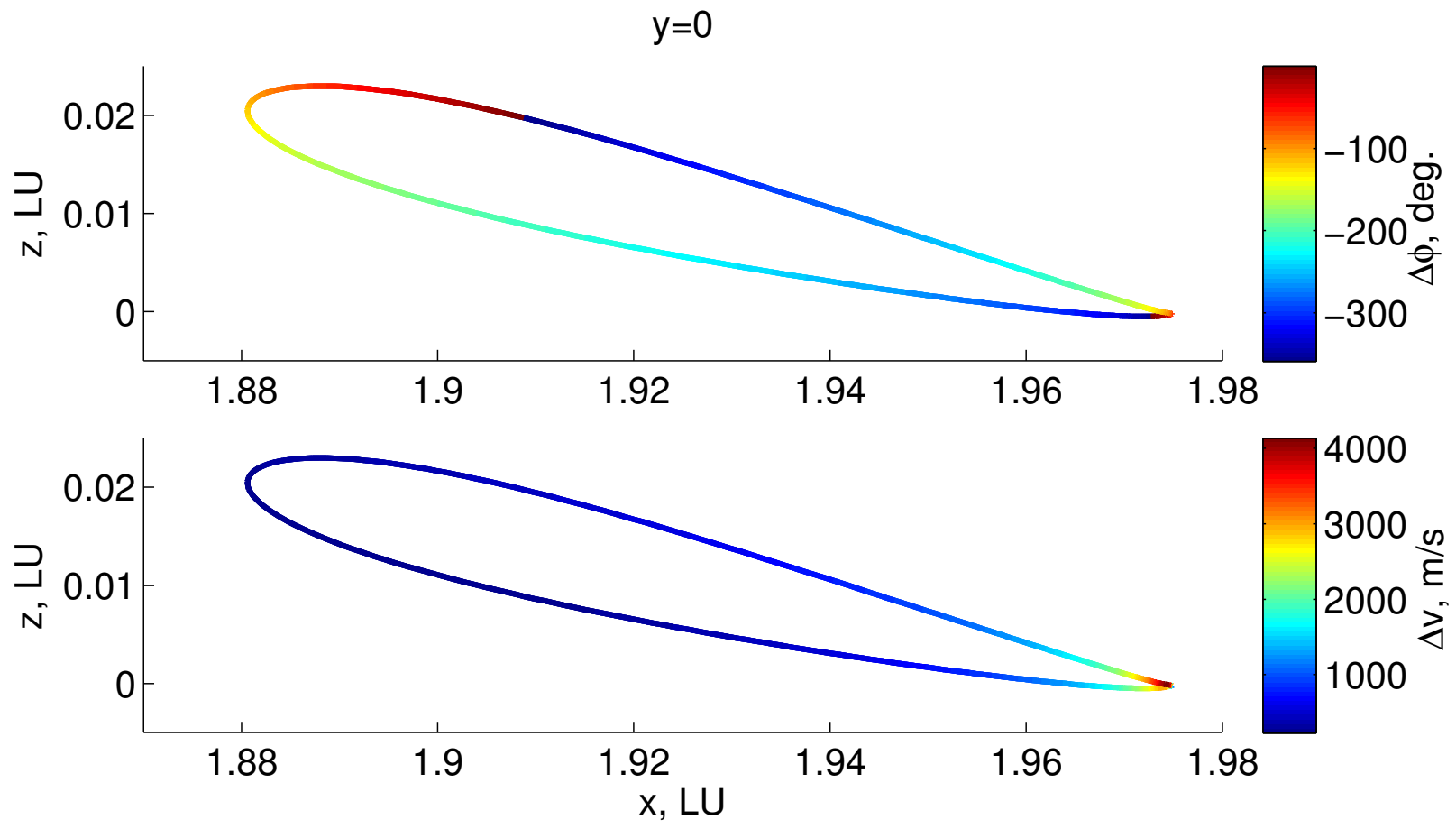

Fig. 7. Manifolds on the Poincare section of $y=0$, along with $\Delta v$ and $\Delta \varphi$. 
Fig. 8 shows the relationship between $\Delta v$ and $\Delta \varphi$. There are two lines because phase angles repeat every period of the halo orbit. The minimum $\Delta v$ is $250 \mathrm{~m} / \mathrm{s}$ at $\Delta \varphi=-180^{\circ}$. The order of the $\Delta v$ for different $\Delta \varphi$ is larger than that of using two-impulsive correction maneuvers. However, since only one $\Delta v$ is applied in this method, $\Delta \varphi$ cannot be freely changed. In other words, $\Delta v$ is naturally associated with $\Delta \varphi$. Some modification is made in the next subsection to gain a flexibility of changing $\Delta \varphi$ as well as the minimum required $\Delta v$.

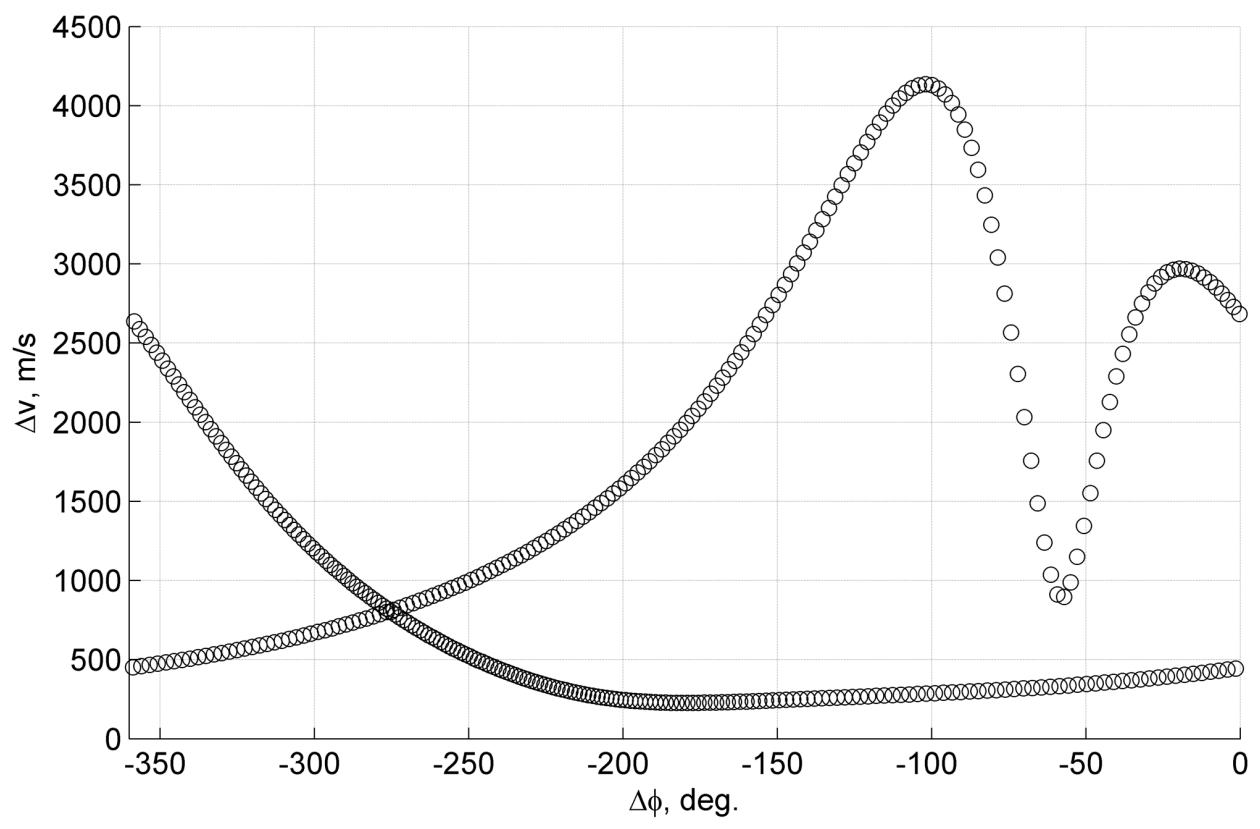

Fig. 8. $\Delta v$ vs $\Delta \varphi$ for patched manifold trajectories.

\section{c. Symmetric connecting trajectories}

Neither the correction maneuvers applied in the transfer phase prior to the halo orbit, nor the manifold patch on the Moon side can achieve the desired configuration with affordable $\Delta v$. To take advantage of the lunar gravity, the unstable and stable manifolds on the Moon side are still used in this subsection. Recalling the symmetry property, if a $\Delta \mathbf{v}_{1}$ (i.e., $\left[\Delta v_{x}, \Delta v_{y}, \Delta v_{z}\right]^{\mathrm{T}}$ ) is placed along the Moon-ward unstable manifold before an $x$ - $z$ plane crossing, such that $v_{x}$ and $v_{z}$ will be zero at the crossing, the resulting trajectory after the crossing will mirror the trajectory before, and hence goes back to the stable manifold as well as the. Another $\Delta \mathbf{v}_{2}$ (i.e., $\left[-\Delta v_{x}, \Delta v_{y},-\Delta v_{z}\right]^{\mathrm{T}}$ ) that is symmetric to $\Delta \mathbf{v}_{1}$ is needed for the insertion to the stable manifold trajectory that is also symmetric to the departure unstable manifold trajectory. 
Recalling Eq. (10), by varying the flight time (i.e. $T o F / 2$ ) to arrive at $\left[v_{x}, v_{z}, y\right]^{\mathrm{T}}=[0,0,0]^{\mathrm{T}}, \Delta \varphi$ can be altered. For a specified $\Delta \varphi$, the optimization program is to find the independent variables, namely, $\tau_{0}$ of the departure manifold trajectory, $t_{1}$ when $\Delta \mathbf{v}_{1}$ is placed, and $\Delta \mathbf{v}_{1}$, such that $v_{x}, v_{z}$ and $y$ are zero $T o F / 2$ after leaving the halo orbit and $\Delta v_{1}$ is a minimum. Because $\Delta v_{1}$ is minimized, the total $\Delta v$ (i.e. $\Delta v_{1} \times 2$ ) is also minimized. Again, the optimization is done with the MATLAB fmincon routine. Multiple initial guesses of $\tau_{0}$ and $t_{1}$ are tried to ensure a global optimality.

Fig. 9 shows the minimum $\Delta v$ vs $\Delta \varphi$. The figure exhibits several Pareto fronts. By investigation, they are found associated with different types of trajectories. When $\Delta \varphi$ is close to 0 or $\pm 360^{\circ}$, the optimal phasing trajectories stay close to the halo orbit, as indicated in the figure. This type of trajectory is referred to as Type 1 . The $\Delta v$ associated with Type 1 increases readily with $|\Delta \varphi|$ and $360^{\circ}-|\Delta \varphi|$. The trajectories touring the Moon (Type 2) require lower $\Delta v$ for larger $|\Delta \varphi|$ and $360^{\circ}-|\Delta \varphi|$. For Type 2, the minimum $\Delta v$ is generally above $100 \mathrm{~m} / \mathrm{s}$. Results in Fig. 8 are found within the interval $T o F<50$ days. If the maximum $T o F$ is extended by one period of the halo orbit, the same $\Delta \varphi$ recur. Longer flight time suggests that a small $\Delta v$ can make an influential difference. Fig. 10 shows the results within the interval $T o F \in[50,75]$ days. It can be seen that, with additional flight time, optimal trajectories can reach to as far as $L_{1}$ (Type 3). Type-3 trajectories can achieve a wide range of $\Delta \varphi$ with $\Delta v$ smaller than $100 \mathrm{~m} / \mathrm{s}$. In addition, Figs. 9,10 show disconnected profiles for Type 2. These different profiles are associated with different types of Type-2 trajectories; namely, different numbers of revolutions about the Moon and EML2.

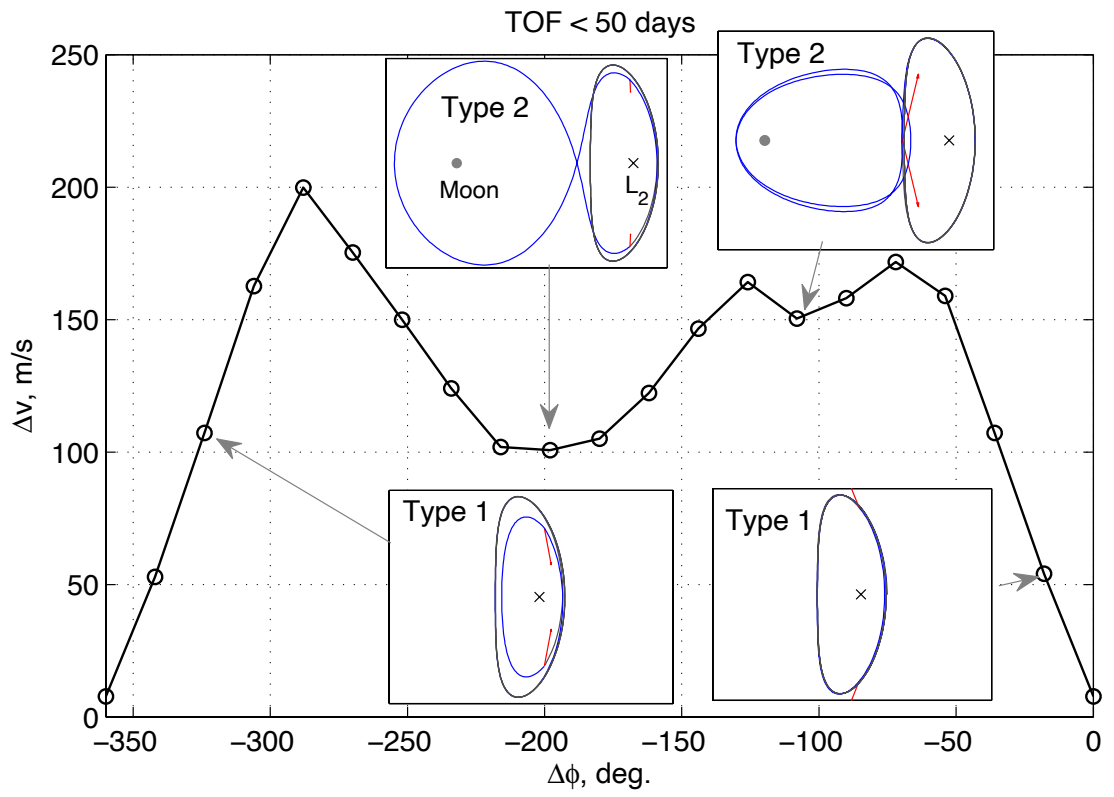

Fig. 9. Minimum $\Delta v$ vs $\Delta \varphi$ for the phasing using symmetric connecting trajectories with $T o F<50$ days. 


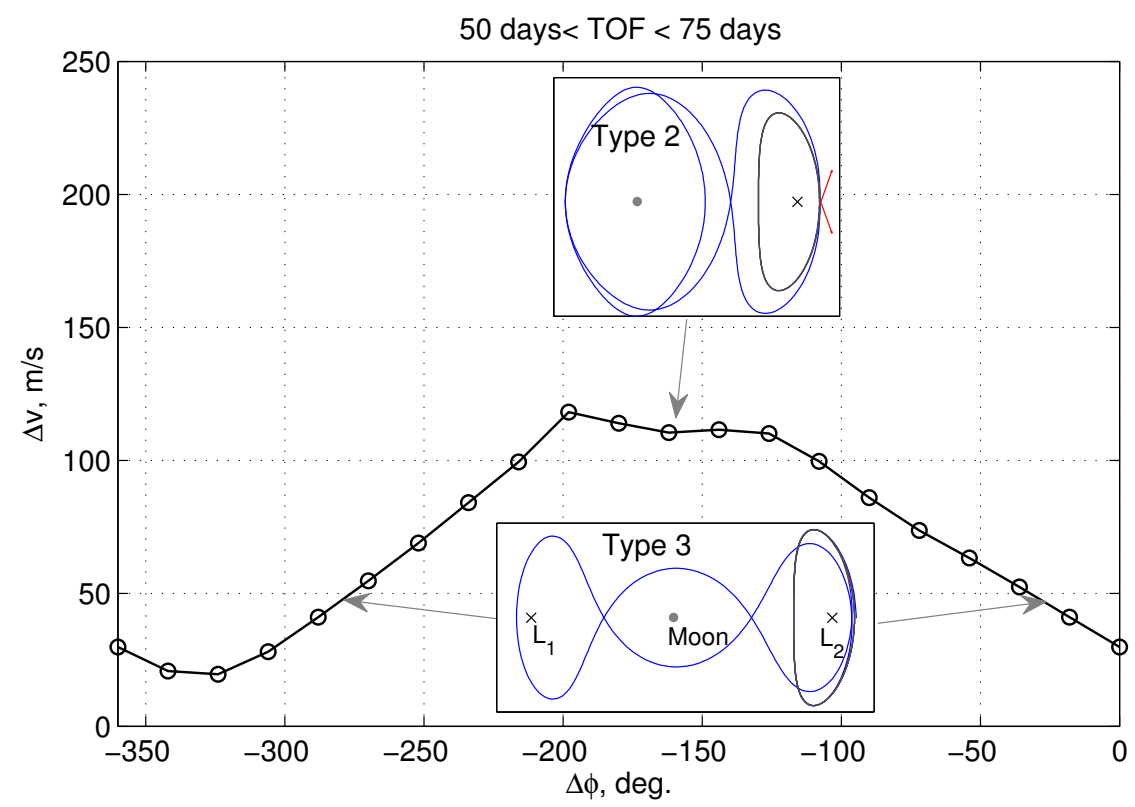

Fig. 10. Minimum $\Delta v$ vs $\Delta \varphi$ for the phasing using symmetric connecting trajectories with $T o F \in[50,75]$ days.

To achieve even distribution with minimized required $\Delta v$ for one CubeSat, it is decided to deploy the four CubeSats to the phase angles that are $-20^{\circ},-110^{\circ},-200^{\circ}$ and $-290^{\circ}$ from the initial orbit. The $\Delta v$ are $55,99,101$ and $42 \mathrm{~m} / \mathrm{s}$, respectively (see Fig. 9 and Fig. 10). Hence, the maximum required $\Delta v$ for one CubeSat is $101 \mathrm{~m} / \mathrm{s}$. Fig. 11 shows the corresponding phasing trajectories. As demonstrated, starting from the same point in the halo orbit at time $t=0$, the four CubeSats are distributed evenly along the halo orbit in 58 days. 


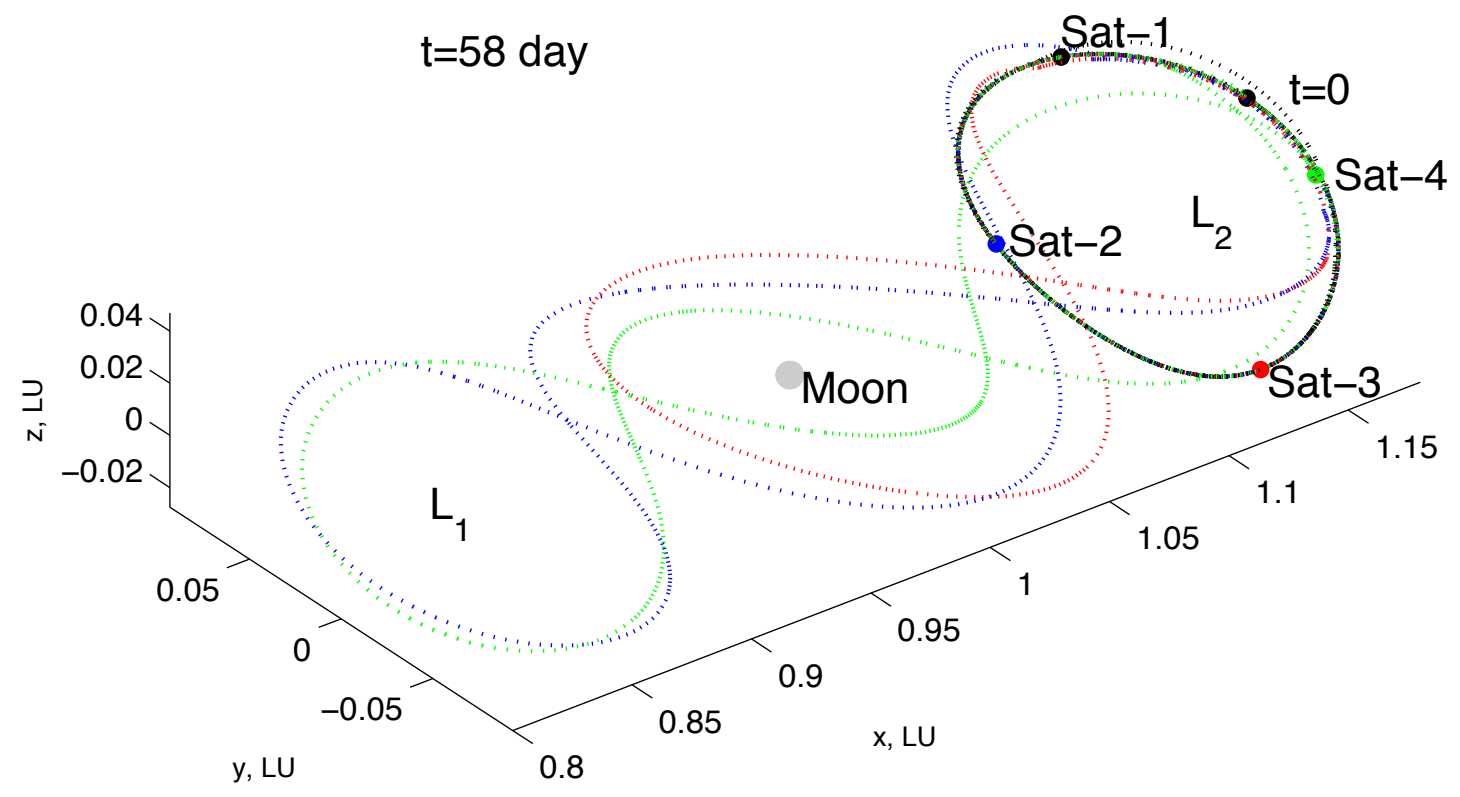

Fig. 11. Phasing trajectories (dashed) to deploy four CubeSats evenly along the halo orbit (solid black).

\section{d. Discussions}

By investigation, it is found that a cold gas propulsion system for CubeSats with a mass of $3.5 \mathrm{~kg}$ (estimated propellant mass around $1.93 \mathrm{~kg}$ ) can provide a total impulse of $750 \mathrm{~N}-\mathrm{sec}^{\dagger}$. The designed PHOEBE CubeSat without a propulsion system has a mass of $4.7 \mathrm{~kg}$. Consequently, a $\Delta v$ budget of $105 \mathrm{~m} / \mathrm{s}$ is available, which can meet the requirement of deployment.

The method of forming symmetric connecting trajectory can be regarded as a special case of the two-impulsive correction method. The specialty is that it takes advantage of the symmetry property and lunar gravity to simplify the trajectory design. The symmetric connecting trajectory is similar to the "bridging trajectory" demonstrated by Davis et al. [9] in the sense that they both connect unstable and stable manifold trajectories. The difference is that the symmetric connecting trajectory can be used for the transfer starting and arriving at the same libration-point orbit, while the bridging trajectory can be used for the transfer between a $L_{1}$ orbit and an $L_{2}$ orbit. In addition, the process of deployment is reverse to the process of rendezvous. Sato et al. [16] have optimized low-thrust trajectories for the

\footnotetext{
${ }^{\dagger}$ http://www.cubesat-propulsion.com/jpl-marco-micro-propulsion-system/, accessed October 22016.
} 
rendezvous of two spacecraft in the same halo orbit. While they did not impose the constraint of symmetry, their optimal trajectories for large initial $\Delta \varphi$ also tour the Moon and exhibit symmetry about the $x$-z plane. That implies the optimal symmetric trajectories solved in the previous subsection are of global optimality, and the present method can be applied to rendezvous missions as well.

Although the Earth-Moon CR3BP is a simplified model without considering the eccentricity of Moon's orbit and other perturbations such as solar radiation pressure, the conclusions obtained in this model are considered reliable to the real world. The presented method can be applied to high-fidelity models with minor modification (usually through correction $\Delta v$ ).

\section{V.Conclusion}

It is desirable to deploy four CubeSats along an Earth-Moon $L_{2}$ halo orbit to provide positioning service for users on the far side of the Moon. In this Note, the deployment trajectories that apply two-impulsive correction maneuvers, patched manifolds and symmetry of the three-body problem have been investigated. The result indicates that the combination of manifolds and symmetric connecting trajectories can lead to various phase angle differences with relatively low $\Delta v$. These phasing trajectories can be used for forming constellations in a halo orbit. It is obtained that a $\Delta v$ around $100 \mathrm{~m} / \mathrm{s}$ is needed to deploy four CubeSats evenly along the halo orbit (corresponds to a positioning accuracy of $1 \mathrm{~km}$ on the surface of the Moon). Three types of phasing trajectories, namely, around the halo orbit, touring the Moon and touring the Earth-Moon $L_{1}$ point, are involved in the deployment. A modern CubeSat propulsion system can meet the $\Delta v$ requirement. Moreover, this phasing method can be applied to not only constellation deployment, but also rendezvous in a three-body system.

\section{Acknowledgments}

This work is supported by the National Natural Science Foundation of China (grant 11372311) and the Advanced Study Program (grant CSU-QZKT-201711) of Technology and Engineering Center for Space Utilization, Chinese Academy of Sciences. The authors wish to thank Yang Gao for the support of the PHOEBE project, Wenbin Wang and Jiangkai Liu for their comments on navigation problems.

\section{References}

[1] Chen, H., Meng, Y., and Ma, J., "Phasing Trajectories for a CubeSat Lunar Far-side Positioning Mission”, 
26th Workshop on Astrodynamics and Flight Mechanics, ISAS/JAXA, Jul. 25-26, 2016.

[2] Burns, J. O., Kring, D. A., Hopkins, J. B., Norris, S., Lazio, T. J. W., and Kasper, J., “A Lunar L2-Farside Exploration and Science Mission Concept with the Orion Multi-Purpose Crew Vehicle and a Teleoperated Lander/rover," Advances in Space Research, vol. 52, 2013, pp. 306-320. doi:10.1016/j.asr.2012.11.016

[3] Li, F., Zhange, H., Wu, X., Ma, J., and Zhou, W., “The Science Value and Technical Challenge of Chang'E4 Landing on the Far-side of the Moon," 41st COSPAR Scientific Assembly, Abstract No. B0.1-18-16, Istanbul, Turkey, July 2016.

[4] Hill, K., and Born, G. H., “Autonomous Interplanetary Orbit Determination Using Satellite-to-Satellite Tracking," Journal of Guidance, Control, and Dynamics, vol. 30, 2007, pp. 679-686. doi:10.2514/1.24574

[5] Hesar, S. G., Parker, J. S., Leonard, J. M., McGranaghan, R. M., and Born, G. H., "Lunar far side surface navigation using Linked Autonomous Interplanetary Satellite Orbit Navigation (LiAISON)," Acta Astronautica, vol. 117, 2015, pp. 116-129. doi:10.1016/j.actaastro.2015.07.027

[6] Kuppers, M., Carnelli, I., Galvez, A., Mellab, K., Michel, P., and the AIM team, "The Asteroid Impact Mission (AIM)”, European Planetary Science Congress, Abstract No. EPSC2015-162, 2015. http://meetingorganizer.copernicus.org/EPSC2015/EPSC2015-162-1.pdf

[7] Koon, W.S., Lo, M. W., Masden, J.E., and Ross, S.D., "Low Energy Transfer to the Moon”, Celestial Mechanics and Dynamical Astronomy, vol. 81, 2001, pp.63-73.

[8] Gómez, G., Koon, W.S., Lo, M.W., Marsden, J.E. Ross, S.D., "Connecting orbits and invariant manifolds in the spatial restricted three-body problem", Nonlinearity, vol. 19, no. 5, 2004, pp. 1571-1606. doi: $10.1088 / 0951-7715 / 17 / 5 / 002$

[9] Davis, K.E., Anderson, R.L., Scheeres, D.J., and Born, G.H., "The Use of Invariant Manifolds for Transfers Between Unstable Periodic Orbits of Different Energies", Celestial Mechanics and Dynamical Astronomy, vol. 107, 2010, pp. 471-485. doi: 10.1007/s10569-010-9285-3

[10] Folta, D., Dichmann, D., Clark, P., Haapala, A. F., and Howell, K., "Lunar Cube Transfer Trajectory Options," AAS/AIAA Space Flight Mechanics Meeting, Williamsburg, VA, Jan. 11-15, 2015, Paper No. AAS 15-353, Advances in the Astronautical Sciences, vol. 155, Furfaro, R., Casotto, S., Trask, A. and Zimmer, S. (ed.), San Diego, Univelt, 2015.

[11] Mathur, R., "Low Thurst Trajectory Design and Optimization: Case Study of a Lunar CubeSat Mission," 6th 
International Conference on Astrodynamics Tools and Techniques, Darmastadt, Germany, Mar. 14-17, 2016. https://indico.esa.int/indico/event/111/session/21/contribution/150/material/paper/0.pdf

[12] Szebehely, V., Theory of Orbits: The Restricted Problem of Three Bodies, New York, San Francisco, London, Academic Press, 1967, pp. 134-139.

[13] Howell, K. C., “Three-Dimensional, Periodic, 'Halo’ Orbits,” Celestial Mechanics, vol. 32, 1984, pp. 53-71.

[14] Gomez, G., Howell, K., Masdemont, J., and Simo, C., "Station-keeping strategies for translunar libration point orbits", AAS/AIAA Astrodynamics Specialist Conference, Monterey, California, Feb. 9-11, 1998, Paper No. AAS 98-168, Advances in the Astronautical Sciences, vol. 99, Middour, J. W., Sackett, L.L., D'Amario, L., and Byrnes, D. V. (ed.), Univelt, 1998, pp. 949-967.

[15] Woodard, M. A., Cosgrove, D., Morinelli, P., Marchese, J., Owens, B., and Folta, D. C., and "Orbit Determination of Spacecraft in Earth-Moon L1 and L2 Liberation Point Orbits," AAS/AIAA Astrodynamics Specialist Conference, August 1-4, 2011, Girdwood, Alaska, Paper No. AAS 11-514, Advances in the Astronautical Sciences, vol. 142, Schaub, H., Gunter, B. C., Russell, R. P., and Cerven, W. T. (ed.), San Diego, Univelt, 2012, pp. 1701-1714.

[16] Sato, Y., Kitamura, K., and Shima, T., "Spacecraft Rendezvous Utilizing Invariant Manifolds for a Halo Orbit", Transactions of the Japan Society for Aeronautical and Space Sciences, vol. 58, No. 5, 2015, pp. 261269. 